\title{
Editorial
}

\section{Reliable Communications over Rapidly Time-Varying Channels}

\author{
Geert Leus, ${ }^{1}$ Georgios Giannakis, ${ }^{2}$ Jean-Paul Linnartz, ${ }^{3}$ Xiaoli Ma, ${ }^{4}$ Ananthram Swami, ${ }^{5}$ and \\ Cihan Tepedelenlioğlú 6 \\ ${ }^{1}$ Faculty of Electrical Engineering, Mathematics, and Computer Science, Delft University of Technology, Mekelweg 4, \\ 2628CD Delft, The Netherlands \\ ${ }^{2}$ Department of Electrical and Computer Engineering, University of Minnesota, Minneapolis, MN 55455, USA \\ ${ }^{3}$ Philips Research, 5656 AA Eindhoven, The Netherlands \\ ${ }^{4}$ School of Electrical and Computer Engineering, Georgia Institute of Technology, Atlanta, GA 30332, USA \\ ${ }^{5}$ Army Research Lab., 2800 Powder Mill Road, Adelphi, MD 20783-1197, USA \\ ${ }^{6}$ Department of Electrical Engineering, Arizona State University, Tempe, AZ 85287, USA
}

Received 17 September 2006; Accepted 17 September 2006

Copyright (C) 2006 Geert Leus et al. This is an open access article distributed under the Creative Commons Attribution License, which permits unrestricted use, distribution, and reproduction in any medium, provided the original work is properly cited.

Wireless communications have become an important part of everyday life. Think for instance about mobile telephone applications, wireless local area networks (WLANs), and wireless ad hoc networks. Most of these systems have been designed assuming that the channel can be regarded as constant over a block of data. Nonetheless market studies predict a rapid growth of high data rate mobile applications such as TV broadcast and video streaming and multiperson wireless gaming. In such mobile applications, Doppler shifts introduce temporal channel variations, which become more pronounced as the carrier frequency increases, and basically violate the time-invariance assumption. Further, with high mobility, terrain changes induce rapid changes in the channel response. As a result, many existing wireless systems can only provide low data rates at high mobility (e.g., UMTS) or even break down completely at high speeds (e.g., DVB-T and IEEE802.16).

This special issue therefore focuses on communications over rapidly time-varying channels, which cannot be viewed as time-invariant over a frame. It is intended to gather new and insightful results in this challenging research area that is gaining increasing attention due to its importance in future wireless applications.

Different models have recently been proposed to track time-varying channels, such as the basis expansion model (BEM) and the Gauss-Markov model (GMM). Such channel models can be used to efficiently estimate the unknown time-varying propagation channel. In the first two papers, the authors rely on the complex exponential BEM to develop training-based and semiblind channel estimators. Tugnait et al.exploit superimposed pilots in the first paper, whereas Barhumi et al. exploit time-multiplexed pilots in the second paper. In the third paper by Misra et al., the GMM is considered, and optimal time-multiplexed training is discussed. Channel models like the BEM or the GMM are mainly aimed at modeling the short-term fading. Long-term fading is usually assumed to be constant, but can also be modeled as a stochastic process. This has been studied in the fourth paper by Olama et al. In addition, this paper presents power control strategies that are related to this new stochastic longterm fading channel model.

Serial time-varying equalizers can be adopted to equalize the time-varying channel. They can either be designed based on channel knowledge, or directly estimated by exploiting pilots. In the fifth paper, Tomasin proposes to equalize the time-varying channel by means of a bidirectional time-varying decision feedback equalizer (DFE), consisting of a time-invariant frequency-domain feedforward part and a time-varying time-domain feedback part. The method relies on a linear BEM model for the channel, and shows improved performance over time-invariant methods. If one models the channel by means of a complex exponential BEM, the complexity of equalizer design can often be reduced by structuring the equalizer also as a complex exponential BEM. Equalizer design in this context has been discussed in the second paper as well as in the sixth paper by 
Verde. Another approach that can be taken is a so-called variable burst transmission approach, in which the burst size over which the channel is assumed to be constant is changed according to the variation of the channel. Assuming limited feedback from the receiver to the transmitter, one can then also adapt the modulation scheme from burst to burst, depending on the instantaneous signal-to-noise ratio. These are subjects studied in the seventh paper by Bui and Hatzinakos.

In multicarrier transmissions, fast time-variations destroy the orthogonality among carriers and introduce what is known as intercarrier interference (ICI). Simulations show that the ICI is generally limited to neighboring carriers, a feature that can be enforced by appropriate time-domain windowing techniques. Exploiting this property, low-complexity equalization schemes have been developed by Hwang and Schniter in the eighth paper and by Rugini et al. in the ninth paper. Both papers also present training-based channel estimation algorithms exploiting frequency/time-multiplexed pilots. The tenth paper by Mallik and Koetter proposes a generalized multicarrier scheme for time-varying channels with modulating and demodulating functions that are localized in time and frequency. In addition, this paper presents multilevel codes matched to the new modulation scheme.

Multiantenna systems, also known as multiple-input multiple-output (MIMO) systems, have been shown to significantly increase the data rate and/or the performance of a wireless communications system through the use of appropriate coding. However, channel estimation (and thus also data detection) is far more complicated in a MIMO system than in a traditional single-input single-output (SISO) system, and this is accentuated when time-varying channels are involved. That is why channel estimation and data detection over fast fading MIMO channels will become a severe problem in future wireless systems. In this special issue, a few papers already deal with this problem. In the eleventh paper, Şenol et al. propose a training-based channel estimator for space-time and space-frequency coded MIMO multicarrier systems exploiting frequency/time-multiplexed pilots. Note though that in contrast with the multicarrier papers mentioned above, the channel here is assumed to change from multicarrier symbol to multicarrier symbol and to be static within a symbol, that is, no ICI is assumed. Further, Mikhael and Yang propose a purely blind MIMO channel tracker based on independent component analysis (ICA) in the twelfth paper. Finally, a semiblind iterative joint channel estimation and data detection approach for MIMO channels exploiting error-correcting codes and time-multiplexed pilots is presented in the thirteenth paper by Simoens and Moeneclaey. A related approach has been introduced by Schoeneich and Hoeher in the fourteenth paper, but in the context of a code-division multiplexing/multiple-access system. To keep track of the channel, this method relies on error-correcting codes as well as on code-multiplexed pilots.

To conclude, this special issue gives a partial update of the state-of-the-art in the field of wireless communications over rapidly time-varying channels. We hope that the presented results will enable interesting new ways to enjoy the benefits of wireless communications, and that the remaining open problems will inspire researchers to continue or start working in this exciting research area.

Geert Leus

Georgios Giannakis

Jean-Paul Linnartz

Xiaoli Ma

Ananthram Swami

Cihan Tepedelenlioğlu

Geert Leus was born in Leuven, Belgium, in 1973. He received the Electrical Engineering degree and the Ph.D. degree in applied sciences from the Katholieke Universiteit Leuven, Belgium, in June 1996 and May 2000, respectively. He has been a Research Assistant and a Postdoctoral Fellow of the Fund for Scientific Research, Flanders, Belgium, from October 1996 till September 2003. During that period, he was affiliated with

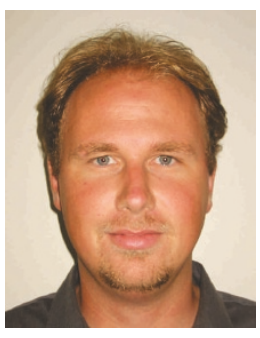
the Electrical Engineering Department of the Katholieke Universiteit Leuven, Belgium. Currently, he is an Assistant Professor at the Faculty of Electrical Engineering, Mathematics and Computer Science of the Delft University of Technology, The Netherlands. His research interests are in the area of signal processing for communications. He received a 2002 IEEE Signal Processing Society Young Author Best Paper Award and a 2005 IEEE Signal Processing Society Best Paper Award. He is a member of the IEEE Signal Processing for Communications Technical Committee, and an Associate Editor for the IEEE Transactions on Signal Processing and the EURASIP Journal on Applied Signal Processing. In the past, he has served on the Editorial Board of the IEEE Signal Processing Letters and the IEEE Transactions on Wireless Communications.

Georgios Giannakis received his Diploma from the National Technical University of Athens, Greece, in 1981; and his M.S. and Ph.D. degrees in 1983 and 1986 from the University of Southern California, all in electrical engineering. Since 1999 he has been a professor with the ECE Department at the University of Minnesota, where he now holds an ADC Chair in wireless telecommunications. His general interests

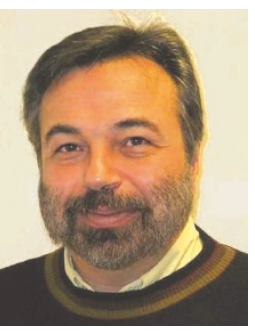
span the areas of communications, networking, and statistical signal processing - subjects on which he has published more than 250 journal papers, 450 conference papers, two edited books, and two research monographs. He is the (co-)recipient of six paper awards from the IEEE Signal Processing (SP) and Communications Societies. He also received Technical Achievement Awards from the SP Society (2000), from EURASIP (2005), a Young Faculty Teaching Award, and the G. W. Taylor Award for Distinguished Research from the University of Minnesota. He has served the IEEE in a number of posts. 
Jean-Paul Linnartz is Senior Director at Philips Research in Eindhoven, the Netherlands, and Department Head of the Connectivity Systems and Networks research group, he is also a part-time professor in the Radio Communication group at Eindhoven University of Technology, where he studies cognitive radio and radio access schemes. He joined Philips in 1995, initially to set up a research activity on conditional access for multimedia content, copy management, digital rights management, and security systems. During 1992-1995, he was an Assistant Professor at The University of California at Berkeley. In 1993, he proposed and analyzed multicarrier CDMA. During 1988-1991 and in 1994, he was Assistant and Associate Professor at Delft University of Technology, respectively. He received his Ph.D. degree (Cum Laude) from T.U. Delft in December 1991 and his M.S. degree (Cum Laude) from Eindhoven University of Technology in 1986. He holds 20 patents and his personal web site is http://ofdm.linnartz.net.

Xiaoli Ma received the B.S. degree in automatic control from Tsinghua University, Beijing, China, in 1998, the M.S. degree in electrical engineering from the University of Virginia, in 2000, and the Ph.D. degree in electrical engineering from the University of Minnesota, in 2003. After receiving her Ph.D. degree, she joined the Department of Electrical and Computer Engineering at Auburn University, where she served

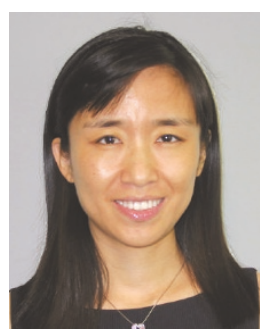
as an Assistant Professor until 2005. Since spring 2006, she has been with the School of Electrical and Computer Engineering at Georgia Tech. Her research interests include transceiver designs for wireless time- and frequency-selective channels, channel estimation and equalization algorithms, carrier frequency synchronization for OFDM systems, routing and cooperative designs for wireless networks.

Ananthram Swami received the B.Tech. degree from IIT, Bombay; the M.S. degree from Rice University, and the Ph.D. degree from the University of Southern California, all in electrical engineering. He has held positions with Unocal, USC, CS-3, and Malgudi Systems. He was a Statistical Consultant to the California Lottery, developed a Matlab-based toolbox for non-Gaussian signal processing, and has held visiting fac-

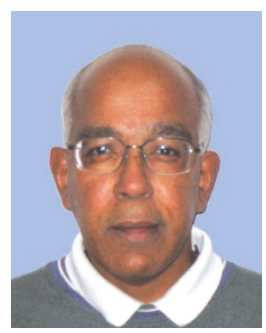
ulty positions at INP, Toulouse. He is currently with the US Army Research Laboratory where his work is in the broad area of signal processing for wireless communications and networking. He is Chair of the IEEE Signal Processing Society's TC on Signal Processing for Communications, an Associate Editor of the IEEE Transactions on Wireless Communications, and of the IEEE Transactions on Signal Processing. He has served as an AE for IEEE Signal Processing Letters, IEEE Transactions on Circuits and Systems-II, and IEEE Signal Processing Magazine. He was coguest editor of a 2004 special issue (SI) of the IEEE Signal Processing Magazine (SPM) on "Signal Processing for Networking," a 2006 SPM SI on "Distributed Signal Processing in Sensor Networks," and a EURASIP JWCN SI on "Wireless Mobile Ad Hoc Networks."
Cihan Tepedelenlioğlu was born in Ankara, Turkey, in 1973. He received the B.S. degree with highest honors from Florida Institute of Technology in 1995, and the M.S. degree from the University of Virginia in 1998, both in electrical engineering. From January 1999 to May 2001 he was a Research Assistant at the University of Minnesota, Minneapolis, where he received the Ph.D. degree in electrical and computer engineer-

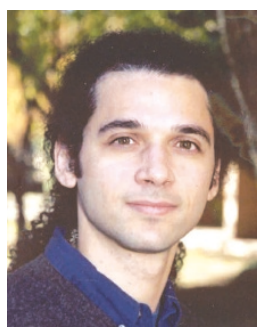
ing. He is currently an Assistant Professor of electrical engineering at Arizona State University. His research interests include wireless communications, estimation, and equalization algorithms for wireless systems, multiantenna communications, filterbanks and multirate systems, OFDM and UWB systems, detection and estimation for sensor networks. He was awarded the NSF (early) Career Grant in 2001. 